\title{
Coexistence of Graves' disease and primary hyperparathyroidism: a case description
}

\author{
Fang Zhang, Xiaohui Pan, Nanwei Tong, Qingguo Lü \\ Division of Endocrinology and Metabolism, Center for Diabetes and Metabolism Research, West China Hospital of Sichuan University, Chengdu, \\ China
}

Correspondence to: Qingguo Lü. Division of Endocrinology and Metabolism, Center for Diabetes and Metabolism Research, West China Hospital of Sichuan University, 37 Guoxue Lane, Chengdu 610041, China. Email: 364167902@qq.com.

Submitted Sep 28, 2021. Accepted for publication Jan 14, 2022.

doi: 10.21037 /qims-21-956

View this article at: https://dx.doi.org/10.21037/qims-21-956

\section{Introduction}

Graves' disease (GD) and primary hyperparathyroidism (PHPT) are two common endocrine disorders. However, patients with coexisting GD and PHPT are seen relatively rarely in the clinic. Since the 1 st case of concomitant hyperthyroidism and hyperparathyroidism was reported in 1936 (1), the coexistence of GD and PHPT has been increasingly recognized by clinicians (2-4). The development of examination methods has made the diagnosis of concurrent GD and PHPT much easier; however, the link between the two disorders remain unclarified. Some researchers have observed that concomitant GD and PHPT is associated with multiple endocrine neoplasia (MEN) $(5,6)$.

We report the case of a 55-year-old woman diagnosed with concomitant GD and PHPT, who presented with a 6-month history of anorexia and weight loss. Due to a failure in immediately localizing the disorder in the parathyroid glands, she first underwent investigations to address her hyper-functioning thyroid. It was not until after she experienced the remission of her hyperthyroidism that a parathyroid adenoma was finally identified as the potential cause of her symptoms, and she became eligible for surgery as further therapy. Preoperative localization is important for decision making of PHPT therapeutic strategy. The experience from this case emphasizes the importance of examination methods and the timely identification of parathyroid adenoma in patients with coexistent GD and PHPT.

\section{Case presentation}

All procedures performed in this study were in accordance with the ethical standards of the institutional and/or national research committee(s) and the Helsinki Declaration (as revised in 2013). Written informed consent was provided by the patient for publication of this case report and accompanying images. A copy of the written consent is available for review by the editorial office of this journal.

A 55-year-old woman was first admitted to our hospital in July 2020 with a 6-month history of anorexia and weight loss. She had been diagnosed 3 months previously with GD at the Chengdu Second People's Hospital, and since then had been taking methimazole. On admission, the patient described having experienced 10 days of palpitations and abnormally excessive sweating consistent with hyperhidrosis. Her family history was unremarkable. Physical examination revealed a body mass index (BMI) of $16.53 \mathrm{~kg} / \mathrm{m}^{2}$. She had mild exophthalmos and a moderate enlargement of the thyroid gland. The thyroid was slightly tender on palpation and moved with deglutition. Her cardiological, pulmonary, abdominal, and neurological evaluations were normal.

Thyroid function evaluation showed the following results: thyroid-stimulating hormone (TSH) $0.025 \mathrm{mU} / \mathrm{L}$ (normal $0.27-4.20 \mathrm{mU} / \mathrm{L}$ ), free triiodothyronine (FT3) $6.63 \mathrm{pmol} / \mathrm{L}$ (normal $3.60-7.50 \mathrm{pmol} / \mathrm{L}$ ), free thyroxine (FT4) $16.10 \mathrm{pmol} / \mathrm{L}$ (normal 12.0-22.0 pmol/L), and thyrotrophin receptor antibody (TRAb) $>40.00$ IU/L (normal $<1.75 \mathrm{IU} / \mathrm{L})$. Initial laboratory studies revealed elevated levels of parathyroid hormone (PTH) $54.30 \mathrm{pmol} / \mathrm{L}$; 

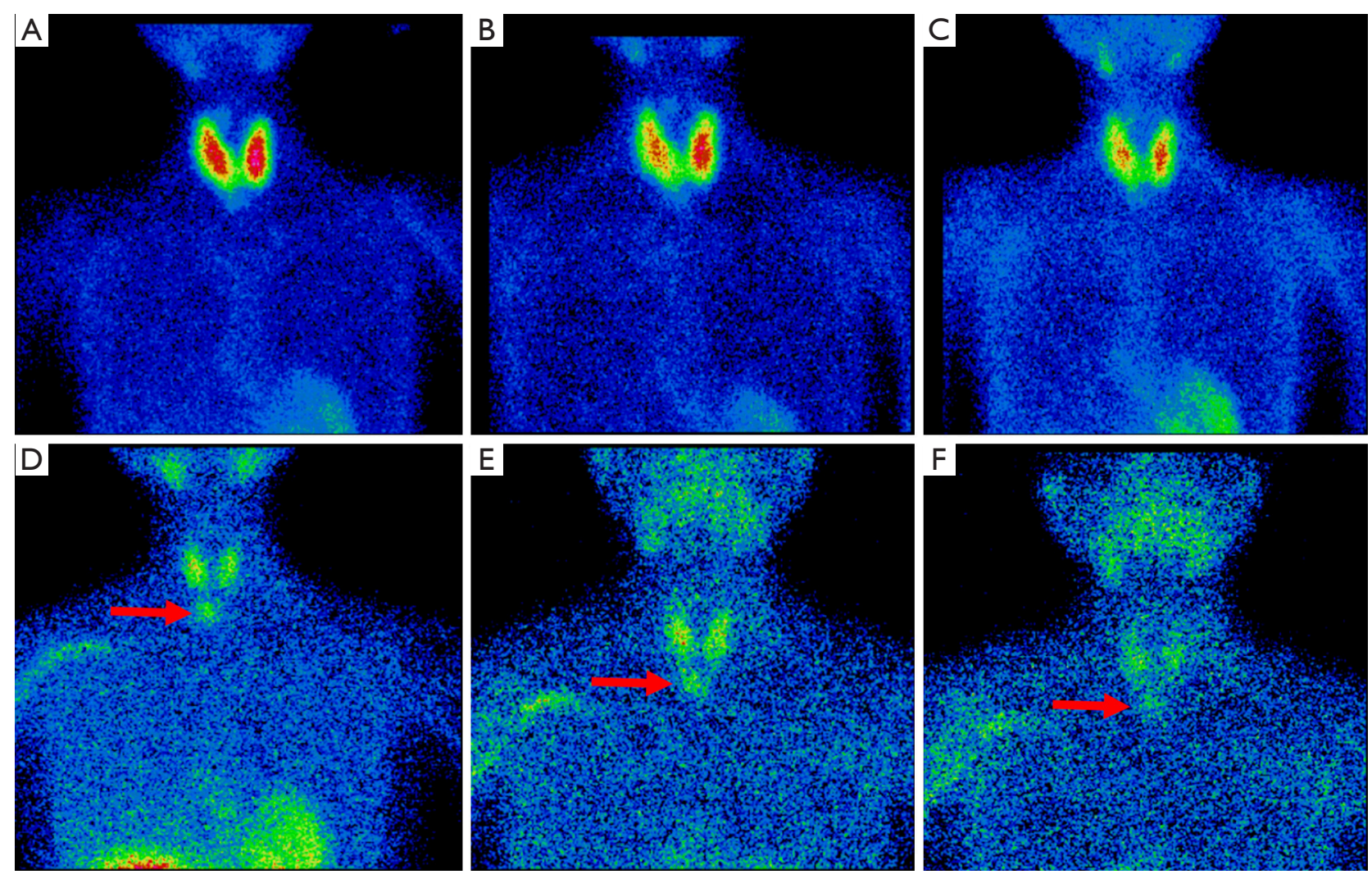

Figure 1 The 99mTc-MIBI scintigraphy images of the patient. (A-C) The first time of MIBI scanning in August 2020: (A) 15 min after 99m Tc-MIBI injection; (B) $1 \mathrm{~h}$ after injection; (C) $2 \mathrm{~h}$ after injection; (D-F) the second time of MIBI scanning in January 2021: (D) 15 min after 99m Tc-MIBI injection; (E) $1 \mathrm{~h}$ after injection; (F) $2 \mathrm{~h}$ after injection. The red arrow indicates the possible lower right parathyroid adenoma. $99 \mathrm{~m}$ Tc-MIBI, technetium-99m-methoxy-isobutyl-isonitrile.

normal $1.60-6.90 \mathrm{pmol} / \mathrm{L})$ and serum calcium $(2.85 \mathrm{mmol} / \mathrm{L}$; normal $2.11-2.52 \mathrm{mmol} / \mathrm{L}$ ), with decreased levels of serum phosphate $(0.84 \mathrm{mmol} / \mathrm{L}$; normal $0.85-1.51 \mathrm{mmol} / \mathrm{L})$ and 25 -hydroxyvitamin D $(32.8 \mathrm{nmol} / \mathrm{L}$; normal $47.7-144.0 \mathrm{nmol} / \mathrm{L})$. The $24-\mathrm{h}$ urinary calcium concentration increased slightly to $8.76 \mathrm{mmol} / 24 \mathrm{~h}$ (normal $2.5-7.5 \mathrm{mmol} / 24 \mathrm{~h}$ ), while the urinary phosphate concentration decreased to $13.25 \mathrm{mmol} / 24 \mathrm{~h}$ (normal 22-48 mmol/24 h). Liver and kidney functions were normal.

The electrocardiogram was normal. Radioactive iodine (RAI) uptake was $48.8 \%$ in the neck at $3 \mathrm{~h}$ (normal 4-8\%) and $71.1 \%$ at $24 \mathrm{~h}$ (normal $8-33 \%$ ). On technetium-99mmethoxy-isobutyl-isonitrile (99m Tc-MIBI) scintigraphy, diffuse enlargement of the bilateral thyroid lobes with enhanced $99 \mathrm{mTc}$ absorption was observed, consistent with hyperthyroidism, but without any parathyroid localization (Figure $1 A-1 C$ ). The bone mineral density (BMD) measurements of the lumbar vertebrae L1-L4, femoral neck, total hip, and the distal third of the radius were 0.749 (T-score -2.8), 0.742 (T-score -3.0), 0.709 (T-score -2.0), and 0.445 (T-score -4.5$) \mathrm{g} / \mathrm{cm}^{2}$, respectively, which were significantly decreased.

The patient underwent RAI (also called I-131) treatment on 18th August 2020. In November 2020 (3 months later), she was diagnosed with hypothyroidism, with the following test results: TSH $78.10 \mathrm{mU} / \mathrm{L}$, FT3 $1.09 \mathrm{pmol} / \mathrm{L}$, and FT4 $1.30 \mathrm{pmol} / \mathrm{L}$. She began to take levothyroxine.

She was re-admitted to our hospital in January 2021 for investigation of persistent hypercalcemia. She denied symptoms of palpitations, polydipsia, polyuria, ostealgia, anorexia, or fatigue. She had experienced an $11 \mathrm{~kg}$ weight gain over the past 5 months and her BMI had increased to $20.57 \mathrm{~kg} / \mathrm{m}^{2}$. Neck examination found a normal thyroid volume. Other physical examination results were the same as before. 

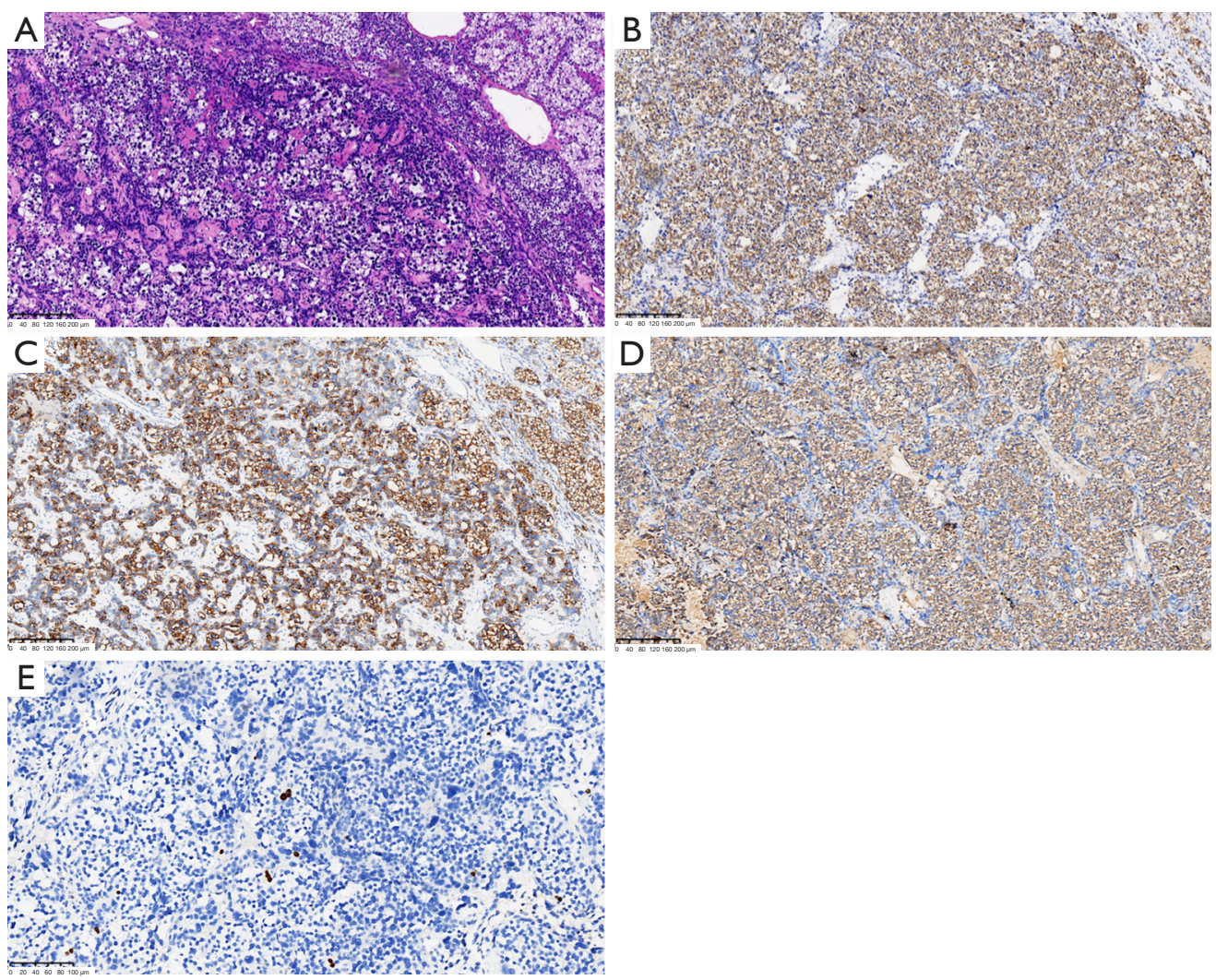

Figure 2 Histological findings of the removed parathyroid adenoma. (A) Representative example of HE staining ( $\times 100$ magnification). Scale bar, $200 \mu \mathrm{m}$; (B) strong expression of PTH on IHC staining ( $\times 100$ magnification). Scale bar, $200 \mu \mathrm{m}$; (C) positive expression of Syn on staining ( $\times 100$ magnification). Scale bar, $200 \mu \mathrm{m}$; (D) strong expression of CgA on staining ( $\times 100$ magnification). Scale bar, $200 \mu \mathrm{m}$; (E) the expression of $\mathrm{Ki}-67$ on IHC staining is up to $1-2 \%$ in the cellular area ( $\times 200$ magnification). Scale bar, $100 \mu \mathrm{m}$. HE, hematoxylin and eosin; PTH, parathyroid hormone; IHC, immunohistochemical; Syn, synaptophysin; CgA, chromogranin A.

The laboratory test results showed PTH $40.84 \mathrm{pmol} / \mathrm{L}$, 25-hydroxyvitamin D $26.0 \mathrm{nmol} / \mathrm{L}$, serum calcium $2.73 \mathrm{mmol} / \mathrm{L}$, and serum phosphate $0.70 \mathrm{mmol} / \mathrm{L}$. Both bone alkaline phosphatase (B-ALP) $37.88 \mu \mathrm{g} / \mathrm{L}$ (normal 11.40-24.60) and serum osteocalcin $48.8 \mathrm{ng} / \mathrm{mL}$ (normal 15.0-46.0 ng/mL) were elevated. Her urinary calcium, phosphate and creatinine were 4.12, 11.70, and $5.31 \mathrm{mmol} / 24 \mathrm{~h}$, respectively. The functions of the liver, kidney, pituitary gland, and adrenal gland, as well as the levels of calcitonin, gastrin, and tumor markers were normal. The cervical ultrasonography did not detect any nodule in, behind, or around the thyroid. The $99 \mathrm{mTc}-\mathrm{MIBI}$ scintigraphy revealed an enhancement of $99 \mathrm{mTc}$ absorption in the lower part of the right parathyroid gland (Figure $1 D-1 F)$, which indicated the possibility of a parathyroid adenoma.

The patient refused to undergo gene detection or gallium-68 positron emission tomography/computed tomography (PET/CT) scan for MEN screening. A surgical exploration was carried out on 25th January 2021. A lower right parathyroid adenoma resection was performed, and the patient was stable after surgery. During the surgery, a solid nodule of size $20 \mathrm{~mm} \times 15 \mathrm{~mm} \times 12 \mathrm{~mm}$ was detected outside the right lower lobe of the thyroid. The pathology report confirmed the nodule was a parathyroid adenoma, with immunohistochemical (IHC) staining positive for PTH, synaptophysin (Syn), chromogranin A (CgA), and Ki$67(1-2 \%)$ and negative for CD56 and carcinoembryonic antigen (CEA) (Figure 2).

The patient recovered well after surgery, and her laboratory findings normalized rapidly. Her PTH level decreased to $5.68 \mathrm{pmol} / \mathrm{L}$. Her serum calcium concentration reached $2.27 \mathrm{mmol} / \mathrm{L}$ a day after the operation and decreased to $2.24 \mathrm{mmol} / \mathrm{L}$ on the $3 \mathrm{rd}$ postoperative day. 

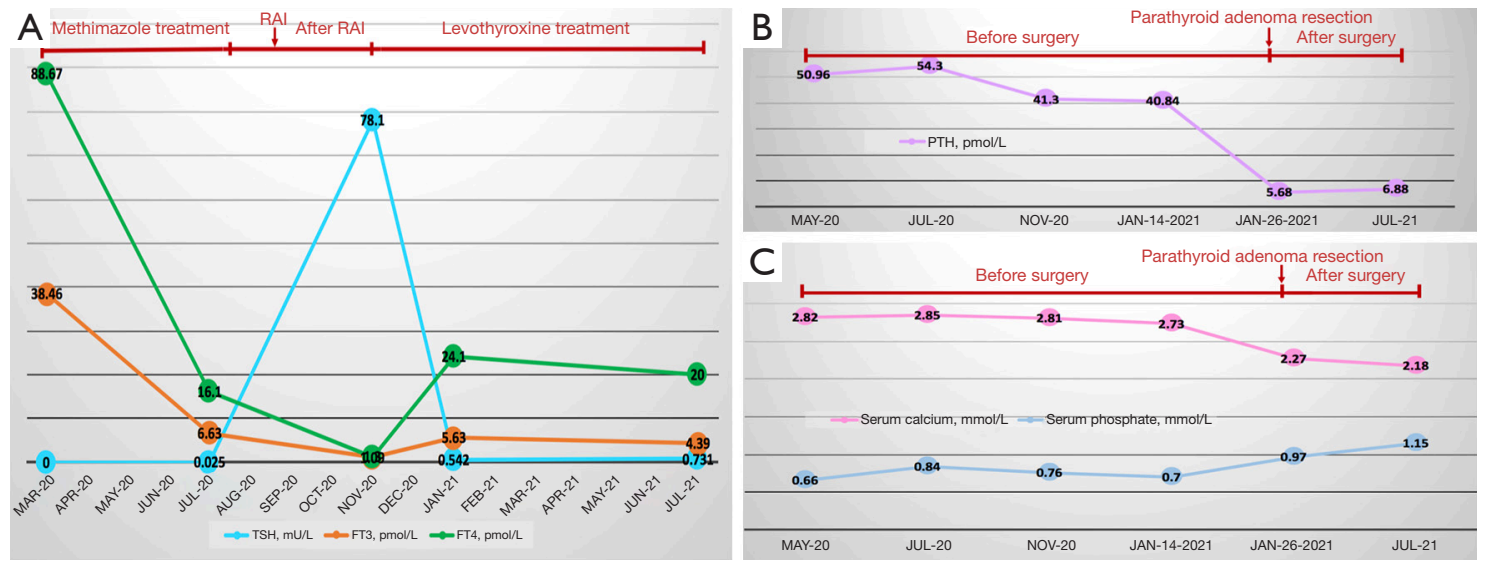

Figure 3 The change of the patient's thyroid and parathyroid functions. (A) The change of thyroid function; (B) the change of parathyroid hormone; (C) the changes of serum calcium and phosphate levels. RAI, radioactive iodine; TSH, thyroid-stimulating hormone; FT3, free triiodothyronine; FT4, free thyroxine; PTH, parathyroid hormone.

The B-ALP and osteocalcin levels decreased to $29.95 \mu \mathrm{g} / \mathrm{L}$ and $36.1 \mathrm{ng} / \mathrm{mL}$, respectively. She received levothyroxine, calcitriol, and calcium pills daily. At 6 months after surgery, the patient had no discomfort. Her serum thyroxin, PTH, and calcium levels remained normal (Figure 3). She still refused to take the MEN gene screening. Follow-up is ongoing.

\section{Discussion}

Concomitant GD in patients with PHPT is now recognized and reported by more and more clinicians. However, sometimes, due to the complicated or non-typical clinical manifestations, the diseases are easily ignored. The patient in our case showed obvious anorexia, weight loss, palpitations, and heat intolerance, and endocrine investigations revealed GD and PHPT. Owing to the patient's hyperthyroid state, $99 \mathrm{~m}$ Tc-MIBI scanning did not immediately localize the disordered parathyroid gland. After remission of the patient's hyperthyroidism by RAI treatment, the parathyroid adenoma was finally detected by 99mTc-MIBI scintigraphy.

The association between thyroid disease and PHPT is now well recognized. Compared with the general population, the occurrence of PHPT is significantly higher in patients with thyroid disease. Vice versa, the incidence of synchronous thyroid abnormalities in patients with PHPT ranges from $16.6 \%$ to $84.3 \%$ (7-9). Sidhu et al. (10) reviewed 65 cases of neck exploration for PHPT and observed 26 cases (40\%) combined with thyroid pathology.
Of these 26 cases, $15 \%$ showed mild multinodular change, $11 \%$ showed severe multinodular change, $4 \%$ showed nodules secondary to Hashimoto's thyroiditis, $5 \%$ were adenomas, and $5 \%$ showed carcinogenic changes. A retrospective study found that the prevalence of coincidental hyperparathyroidism in patients with hyperthyroidism was $13.5 \%$ (2). Recently, a case cohort study summarized that 21 cases with a history of GD developed parathyroid adenoma, where an increased risk of parathyroid adenoma might be possibly linked to the GD patients with a history of RAI treatment, with a mean latency time of 26.2 years (3). Moreover, a retrospective analysis including 469 PHPT cases revealed that patients with a history of RAI treatment presented with similar clinical characteristics to RAInaive PHPT patients, but the risk of recurrence of PHPT might be higher in RAI-exposed patients (11). In our case, although the patient undertook RAI therapy, based on her laboratory findings (Figure 3B,3C) the diagnosis of PHPT was confirmed before commencement of RAI treatment.

Until now, the mechanism of coexistent hyperparathyroidism and hyperthyroidism is still unclear. Some researchers believe the occurrence of this association is just a coincidence, whereas others have advocated that serum calcium level, growth factor, and genetic factors may be involved $(4,5,12,13)$. Further studies are needed to investigate the mechanism between the two disorders and their association.

Although it is rare, concomitant hyperthyroidism and PHPT in a patient should be considered, as should whether these conditions might be the result of MEN. It is well 
known that both MEN type 1 (MEN1) and MEN type 2A (MEN2A) may consist of neoplasia, hyperplasia, or hyperfunction of the thyroid and parathyroid. A clinical practice guideline for MEN1 recommends that genetic testing should be offered to individuals with clinical manifestations of MEN1 (14). Therefore, in our opinion, clinicians should be aware of patients with coexisting hyperthyroidism and hyperparathyroidism that might be caused by MEN, and a MEN screening procedure is recommended. The endocrine laboratory investigation, MEN genetic testing, and gallium-68 PET/CT scanning are useful tools for MEN screening. In our case, growth hormone, insulin-like growth factor 1, prolactin, adrenocorticotropic hormone, cortisol, catecholamine hormone and its metabolites, renin, aldosterone, calcitonin, and gastrin levels of the patient were normal. Enhanced magnetic resonance imaging (MRI) of the patient's adrenal glands was unremarkable. From our perspective, it was necessary to undertake further investigations to find the cause of the patient's condition, however, the patient refused to have the genetic test or gallium-68 PET/CT for MEN identification.

Although the diagnosis of concurrent GD and PHPT is not difficult based on the laboratory findings, preoperative localization is critical for determining the PHPT therapeutic strategy. It is reported that cervical ultrasound has a high positive sensitivity of $83.5 \%$ for predicting parathyroid adenoma, while the $99 \mathrm{mTc}-\mathrm{MIBI}$ scintigraphy has a positive sensitivity of $85 \%$; thus, there is no significant difference between them (15). The diagnostic performance of either PET/CT with 18F-labled choline analogues or 4-dimensional CT is superior to conventional 99mTc-MIBI scintigraphy and neck ultrasound in localizing parathyroid adenoma (16), but neither of them has been carried out in our hospital. The latest practice guideline released by the European Association of Nuclear Medicine recommended combining 99mTc-MIBI scintigraphy with cervical ultrasonography as an acceptable first-line strategy for parathyroid imaging (16). However, ultrasound did not localize any parathyroid gland in this patient, which may have been affected by the location and size of the adenoma. As for the $99 \mathrm{mTc}$-MIBI scan failing to detect any disordered parathyroid adenoma at the first admission, this can be explained by the excessive absorption of $99 \mathrm{mTc}$ by the hyper-functioning thyroid, which completely covered the parathyroid glands. Intriguingly, after the remission of hyperthyroidism, the $99 \mathrm{mTc}-\mathrm{MIBI}$ scintigraphy helped us to confirm the final diagnosis of parathyroid adenoma. This reminds us of the importance of choosing the right method and time to identify the parathyroid adenoma, especially when concurrent complications might interfere with the results.

In conclusion, the co-existence of GD and PHPT may be more prevalent than what has been observed to date. Further studies are needed to clarify the link between the two disorders. Moreover, clinicians should be aware that a MEN screening procedure is recommended in individuals with concomitant hyperthyroidism and PHPT. The present case also emphasizes the importance of examination methods and the timely identification and localization of parathyroid adenoma.

\section{Acknowledgments}

All the authors appreciate the generosity and cooperation of the patient and her family.

Funding: This work was supported by a grant from the 1.3.5 Project for Disciplines of Excellence from the West China Hospital of Sichuan University (No. ZYGD18017).

\section{Footnote}

Conflicts of Interest: All the authors have completed the ICMJE uniform disclosure form (available at https://qims. amegroups.com/article/view/10.21037/qims-21-956/coif). The authors have no conflicts of interest to declare.

Ethical Statement: The authors are accountable for all aspects of the work in ensuring that questions related to the accuracy or integrity of any part of the work are appropriately investigated and resolved. All procedures performed in this study were in accordance with the ethical standards of the institutional and/or national research committee(s) and the Helsinki Declaration (as revised in 2013). Written informed consent was provided by the patient for publication of this case report and accompanying images. A copy of the written consent is available for review by the editorial office of this journal.

Open Access Statement: This is an Open Access article distributed in accordance with the Creative Commons Attribution-NonCommercial-NoDerivs 4.0 International License (CC BY-NC-ND 4.0), which permits the noncommercial replication and distribution of the article with the strict proviso that no changes or edits are made and the original work is properly cited (including links to both the formal publication through the relevant DOI and the license). 
See: https://creativecommons.org/licenses/by-nc-nd/4.0/.

\section{References}

1. Noble JF, Borg JF. Hyperparathyroidism complicated by hyperthyroidism. Report of a case. Arch Int Med 1936;58:846-59.

2. Abboud B, Sleilaty G, Mansour E, El Ghoul R, Tohme C, Noun R, Sarkis R. Prevalence and risk factors for primary hyperparathyroidism in hyperthyroid patients. Head Neck 2006;28:420-6.

3. Wei S, Baloch ZW, LiVolsi VA. Parathyroid adenoma in patients with Graves' disease: a report of 21 cases. Endocr Pathol 2015;26:71-4.

4. Xiao H, Yu B, Wang S, Chen G. Concomitant Graves' disease and primary hyperparathyroidism: the first case report in mainland of China and literature review. Chin Med J (Engl) 2002;115:939-41.

5. He Y, Liu S, Guo H, Shi B. Incidental finding of papillary thyroid carcinoma with BRAFV600E mutation in a patient with coexistent primary hyperparathyroidism and Graves' hyperthyroidism. BMJ Case Rep 2014;2014:bcr2013203436.

6. Bhat S, Davis S. Co-existence of primary hyperparathyroidism due to multiple endocrine neoplasia 1 in a hypercalcemic patient with Graves disease. AACE Clin Case Rep 2019;5:e13-5.

7. Cai X, Yousefzadeh P, Wang X. Hypercalcemia caused by concomitant graves g disease and primary hyperparathyroidism. J Endocrinol Thyroid Res 2018;3:555603.

8. Toursarkissian B, Sloan DA, Schwartz RW. Coexisting hyperthyroidism and primary hyperparathyroidism.

Cite this article as: Zhang F, Pan X, Tong N, Lü Q. Coexistence of Graves' disease and primary hyperparathyroidism: a case description. Quant Imaging Med Surg 2022;12(5):3014-3019. doi: 10.21037/qims-21-956
Surgery 1993;113:716-8.

9. Wright MC, Jensen K, Mohamed H, Drake C, Mohsin K, Monlezun D, Alsaleh N, Kandil E. Concomitant thyroid disease and primary hyperparathyroidism in patients undergoing parathyroidectomy or thyroidectomy. Gland Surg 2017;6:368-74.

10. Sidhu S, Campbell P. Thyroid pathology associated with primary hyperparathyroidism. Aust N Z J Surg 2000;70:285-7.

11. Law RH, Quan DL, Stefan AJ, Peterson EL, Singer MC. Hyperparathyroidism subsequent to radioactive iodine therapy for Graves' disease. Head Neck 2021;43:29943000.

12. Klemm T, Lamesch P, Paschke R. Coincidence of hot thyroid nodules and primary hyperparathyroidism. Exp Clin Endocrinol Diabetes 1999;107:295-8.

13. McGowan DM, Vaswani A, Shperling I. Coexisting hyperparathyroidism with thyrotoxicosis. J Endocrinol Invest 1991;14:305-10.

14. Thakker RV, Newey PJ, Walls GV, Bilezikian J, Dralle H, Ebeling PR, Melmed S, Sakurai A, Tonelli F, Brandi ML; Endocrine Society. Clinical practice guidelines for multiple endocrine neoplasia type 1 (MEN1). J Clin Endocrinol Metab 2012;97:2990-3011.

15. Nasiri S, Soroush A, Hashemi AP, Hedayat A, Donboli K, Mehrkhani F. Parathyroid adenoma Localization. Med J Islam Repub Iran 2012;26:103-9.

16. Petranović Ovčariček P, Giovanella L, Carrió Gasset I, Hindié E, Huellner MW, Luster M, Piccardo A, Weber T, Talbot JN, Verburg FA. The EANM practice guidelines for parathyroid imaging. Eur J Nucl Med Mol Imaging 2021;48:2801-22. 